\title{
Response to Druwe and Burgoon
}

\section{Re: Druwe, I.L. and Burgoon, L.: revisiting Cohen et al. 2015, Cohen et al. 2014 and Waalkes et al. 2014: a bayesian re-analysis of tumor incidences}

\author{
Samuel M. Cohen ${ }^{1} \cdot$ Lora L. Arnold $^{1} \cdot$ James E. Klaunig $^{2} \cdot$ Jay I. Goodman $^{3}$
}

Received: 27 June 2016 / Accepted: 11 August 2016 / Published online: 19 August 2016

(C) Springer-Verlag Berlin Heidelberg 2016

We have determined (Cohen et al. 2014, 2015) that the conclusion, reported by Waalkes et al. (2014), that lung tumors are induced in CD-1 mice by exposure to inorganic arsenic at human-relevant doses is not supported by the data presented. The key reasons for our position are: (1) lack of consistency and reproducibility (Garry et al. 2015); (2) not taking into account the high variability in incidences of lung tumors in control CD-1 mice, including the variability in two studies reported by the Waalkes' group (Tokar et al. 2011; Waalkes et al. 2014); (3) lack of information regarding historical controls; (4) the statistical criterion of significance used by Waalkes et al. does not follow the criterion of Haseman et al. (1986); and (5) the biologic implausibility of the dose response being suggested. When evaluating the incidence of common tumors (background incidence $\geq 1 \%$, which the background incidence of lung tumors in CD-1 mice clearly meets), Haseman et al. (1986) from the National Toxicology Program (NTP) recommended a $p$ value $<0.01$ be utilized for statistical significance, not $p<0.05$ as used by Waalkes et al. (2014). Using this criterion, the mouse lung tumor data reported by Waalkes et al. (2014) are not significant, even using concurrent controls for comparison.

Recently. Druwe and Burgoon (2016) focused on an aspect of the concerns raised by Cohen et al. (2014) and (2015) regarding the variability in lung tumor incidences in control CD-1 mice reported by Tokar et al. (2011) and Waalkes et al. (2014) (it should be noted that they did not

Samuel M. Cohen

scohen@unmc.edu

1 University of Nebraska Medical Center, Omaha, NE, USA

2 Indiana University, Bloomington, IN, USA

3 Michigan State University, East Lansing, MI, USA address the marked variability reported by Charles River Laboratories (Giknis and Clifford 2005), which was referenced by Cohen et al. (2014) and (2015). Druwe and Burgoon (2016) employed Bayesian statistics that included modeling control tumor incidences as Bernoulli distributions and an approach that sets a region of practical equivalence (ROPE) to demarcate a region around zero difference that is functionally equivalent to no difference, to test the null hypothesis that the control tumor incidences from the Tokar et al. (2011) and Waalkes et al. (2014) studies are the same. The null hypothesis was accepted, and the authors concluded that the tumor incidences in both studies are likely from the same distribution. Druwe and Burgoon (2016) next tested the null hypothesis that arsenic exposure does not increase the lung tumor response in male mice. A Bayesian approach was used which involved combining control incidences of lung tumors in CD-1 mice from three studies (Tokar et al. 2011, 2012; Waalkes et al. 2014). Druwe and Burgoon (2016) indicate that their analysis confirms that the Waalkes et al. (2014) study demonstrates low-dose (50 and $500 \mathrm{ppb}$ ) inorganic arsenic increases lung tumor incidences in male CD-1 mice.

We have several concerns with the statistical analyses performed by Druwe and Burgoon: (1) they do not indicate which mathematical formula(s) they used to calculate their Bayesian probabilities nor do they provide a rational for their selection; (2) they do not provide a rational for modeling control tumor incidences as Bernoulli distributions and do not indicate what other distributions were considered and upon what basis(es) they were rejected; and (3) setting of their ROPE appears to be arbitrary in that there is no discussion/consideration of its biological basis; the importance of this point is underlined by Druwe and Burgoon's statement that "Our chief concern with p-values is that they do not represent biological significance." 
Furthermore, in situations where one is evaluating whether or not a chemical treatment caused an increase in a common tumor (background incidence $\geq 1 \%$ ), like lung tumors in CD-1 mice, it is very appropriate to take a more stringent approach as compared to circumstances where rare tumors are involved (Haseman et al. 1986). This makes biological sense and it is unfortunate that the analysis performed by Druwe and Burgoon (2016) ignores this key point. In addition, Druwe and Burgoon do not address any of the other issues raised by Cohen et al. (2014, 2015), in particular for a Bayesian statistical approach, the lack of historical data, not just the 3 studies most recently reported from the Waalkes group.

The discussion presented above reinforces our view expressed previously (Cohen et al. 2014, 2015) that the results reported by Waalkes et al. (2014) show no arsenicrelated lung tumors in CD-1 mice at the low doses administered in these studies.

\section{References}

Cohen SM, Arnold LA, Klaunig JE, Goodman JI (2014) Re: Waalkes et al.: Lung tumors in mice induced by "whole-life" inorganic arsenic exposure at human-relevant doses. Arch Toxicol 88:2061-2062

Cohen SM, Arnold LA, Klaunig JE, Goodman JI (2015) Response to the Waalkes et al., Letter to the editor concerning our "letter to the editor, Re: Lung tumors in mice induced by "whole-life" inorganic arsenic exposure at human relevant doses, Waalkes et al., Arch Toxicol, 2014". Arch Toxicol 89:2167-2168

Druwe IL, Burgoon L (2016) Revisiting Cohen et al. 2015, Cohen et al. 2014 and Waalkes et al. 2014: a bayesian re-analysis of tumor incidences. Arch Toxicol 90(8):2047-2048

Garry MR, Santamaria AB, Williams AL, DeSesso JM (2015) In utero arsenic exposure in mice and early life susceptibility to cancer. Regul Toxicol Pharmacol 73:378-390

Giknis MLA, Clifford CB (2005) Spontaneous neoplastic lesions in the Crl: CD-1 (ICR) mouse in control groups from 18 months and 2 year studies. Charles River Laboratories, Wilmington, pp $1-19$

Haseman JK, Winbush JS, O'Donnell MW Jr (1986) Use of dual control groups to estimate false positive rates in laboratory animal carcinogenicity studies. Fundam Appl Toxicol 7:573-584

Tokar EJ, Bhalchandra AD, Ward JM, Delker DA, Waalkes MP (2011) Carcinogenic effects of "whole-life" exposure to inorganic arsenic in CD1 mice. Toxicol Sci 119:73-83

Tokar EJ, Bhalchandra AD, Waalkes MP (2012) Renal, hepatic, pulmonary and adrenal tumors induced by prenatal inorganic arsenic followed by dimethylarsinic acid in adulthood in CD1 mice. Toxicol Lett 209:179-185

Waalkes MP, Qu W, Tokar EJ, Kissling GE, Dixon D (2014) Lung tumors in mice induced by "whole-life" inorganic arsenic exposure at human-relevant doses. Arch Toxicol 88:1619-1629 